Review Article

\title{
The Effects of Mediterranean Diet on the Human Gut Microbiota; a Brief Discussion of Evidence in Humans
}

\author{
Anita H Kelleher, Angelos K Sikalidis *
}

Department of Food Science and Nutrition, California Polytechnic State University, San Luis Obispo, CA 93407, USA; E-Mails: ahkelleh@calpoly.edu; asikalid@calpoly.edu

* Correspondence: Angelos K Sikalidis; E-Mail: asikalid@calpoly.edu

Academic Editor: Rostyslav Bubnov

Special Issue: Gut Microbiota and Gut Health

OBM Hepatology and Gastroenterology

2021, volume 5 , issue 1

doi:10.21926/obm.hg.2101056
Received: November 09, 2020

Accepted: February 02, 2021

Published: February 05, 2021

\begin{abstract}
The Mediterranean diet (MD) is considered one of the healthiest dietary patterns due to its rich provision of phytochemicals, antioxidants, vitamins, fiber, polyunsaturated, omega-3, and short-chain fatty acids through a variety of foods. The supply of such nutrients and bioactive components can support gut health and reduce systemic inflammation, with accumulating evidence from several human studies demonstrating the utility of the Mediterranean diet in the prevention of chronic and metabolic diseases. Further studies are needed to explore the role of the Mediterranean diet protecting against such diseases and the related mechanisms, including the interplay between components of the MD and gut microbiota. This brief systematic review specifically explores the recent evidence in humans investigating the link between MD and the human microbiota. Herein, over 50 articles were revised and referenced, after a careful vetting process, to produce this manuscript. Articles were ultimately selected based upon the detail and novelty of their content and contribution to the field.
\end{abstract}

\section{Keywords}

Mediterranean diet; gut microbiota; microorganisms; Western diet; bioactive compounds

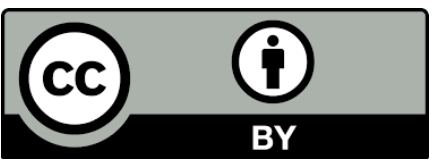

(C) 2021 by the author. This is an open access article distributed under the conditions of the Creative Commons by Attribution License, which permits unrestricted use, distribution, and reproduction in any medium or format, provided the original work is correctly cited. 


\section{Introduction}

Mounting evidence demonstrate the relationship between positive health outcomes and gut microbiome status [1]. A healthy gut microbiome is comprised of a favorable microbial profile and robust diversity, while phenotypically portrays as regular stool frequency and consistency of defecations, lack of gastrointestinal pain and discomfort with a relatively low intensity of gastrointestinal symptoms. An unhealthy gut, also referred to as the state of "dysbiosis", is characterized by a pro-inflammatory condition, a hallmark of a variety of chronic diseases. This state of imbalance can be provoked by a sudden and/or significant change in diet, antibiotic therapies undermining gut ecosystem diversity, caesarean deliveries, excessive hygiene, stress, or a lack of exercise, and often leads to improper immune function [2]. Nonetheless, diet remains one of the strongest predicting factors for long-term gut health [3]. Several studies have examined the effect of different dietary patterns on the microbiota and of particular interest is the Mediterranean diet (MD), due to its otherwise demonstrated positive health outcomes produced, especially as related to metabolic health. Consumption of the MD has been shown to drastically affect the gut microflora and is associated with reduced risk of metabolic disease such as cardiovascular disease (CVD), type 2 diabetes mellitus (T2DM), obesity, insulin resistance, inflammatory disease, neuro-degenerative disease and cancer $[2,4,5]$. The Healthy Mediterranean-Style Eating pattern is one of the dietary strategies recommended in the USDA's 2015-2020 Dietary Guidelines for Americans in an effort to prevent chronic diseases and promote overall health $[4,6]$.

The MD is characterized by high-level intake of fruit, vegetables, nuts, legumes, complex carbohydrates, low intake of saturated fat, red meat, and dairy products, moderate consumption of fish, and moderate to regular consumption of alcohol (principally red wine), with the main source of dietary lipids from olive oil and monounsaturated fats [7]. Complex undigestible carbohydrates require bacterial fermentation and subsequently support healthy microbial activity, which results in the production of bioactive short-chain fatty acids (SCFA), including acetate, butyrate and propionate. SCFA quantification has been used in many studies as a way of determining microbial metabolic activity [2]. The fruits and vegetables provide phytosterol, antioxidant and anti-inflammatory bioactive compounds, while the regular consumption of nuts, legumes, fish and the use of olive oil all provide a considerable amount of unsaturated and omega3 fatty acids. Research has shown that gastric emptying rate for meals enriched in omega- 3 unsaturated fatty acids is significantly higher than that of meals rich in saturated fat, monounsaturated fatty acids or omega-6 fatty acids [8]. Regular wine consumption (especially red) provides a good source of a wide range of polyphenols, specifically anthocyanins, and is associated with a reduced risk for cardiovascular disease $[9,10]$. The composition of the MD is very different from the foods which comprise the Western diet, characterized by an excessive intake of energydense foods rich in predominantly saturated fat, cholesterol, animal protein, refined simple sugars, sodium, and ultra-processed foods. The Western diet, also sometimes referred to as "The American Diet," arguably promotes an inflammatory state and has been shown to increase risk for noncommunicable diseases of metabolic nature [11]. Furthermore, evidence points to the notion 
that the food typical of a Westernized lifestyle may cause the depletion of bacteria taxa that are important for optimal gut function and inflammatory disease prevention, resulting in lower bacterial diversity and different microbial profiles compared to MD consumption [12, 13]. Today, an estimated $39.8 \%$ of the US population is considered obese as assessed by Body Mass Index (BMI) classification system (BMI > 30), while obesity prevalence is steadily increasing to what is often referred to as epidemic proportions $[1,14]$.

\section{Methodology}

In the systematic review presented herein, the recent evidence on the relationship between $\mathrm{MD}$ and the gut microbiota is discussed considering predominantly human studies. After careful scrutiny of over 100 articles, 52 were selected for final revisions based upon the novelty of their findings or analysis, the degree of detail included and the relevance of the topic with the theme of this manuscript. Each article was obtained through either PubMed/Medline or Google Scholar and a ceiling was established so that each article was written within the past three decades. The subsections of the manuscript were established first and the search-words followed specific to each section.

\section{Key Microorganisms in the Gut}

The human gut microbiota comprises the natural niche to trillions of bacteria residing in the large intestine habitat. Researchers have relied on 16S rRNA gene sequencing to identify over 35,000 bacterial species and over 10 million non-redundant genes [1, 15]. As numerous studies have shown, the diversity and microbial richness is highly dependent upon dietary pattern. The Mediterranean diet, vegetarian diets and vegan diets tend to foster the proliferation of different bacterial distributions compared to the "Western" diet [4].

A study carried out in Italy, by Francesca De Filippis et al., assessed gut microbiota and metabolome function after 7 days of strictly following either a vegan, vegetarian, or omnivore diet. The majority of those on the plant-based diets, and 30\% of those assigned the omnivore diet had high adherence to a MD regimen. Among those on plant-based diets, Prevotellaceae abundance increased, though not statistically significantly after accounting for multiple factors. However, an association between the abundance of Prevotella and fruit/vegetable intake was determined. Vegans and vegetarians also demonstrated a higher abundance for the Bacteroidetes phylum and a lower Firmicutes: Bacteroidetes ratio, compared with omnivore diet participants. These latter two types of bacteria are able to degrade carbohydrates not naturally digestible by the host. Additionally, Lachnospira and Prevotella were significantly linked to the high and medium quartiles of the Healthy Food Diversity (HFD) Index used in the study as a health promoting metric, and both were linked to plant-based diets. Interestingly, "Lachnospira is one of the core genera of the gut microbiota and recognized as the only Firmicutes able to degrade pectin beyond Faecalibacterium and Eubacterium [16]." Current research efforts are aimed at exploring why a decrease in Lachnospira abundance is seen consistently in the gut microbiota of subjects with cholesterol gallstones, as well as in the rectal mucosa of HIV patients [16]. Researchers hypothesize it could serve as a biomarker for predisposal to diabetes and other metabolic diseases [16]. Conversely, L-Ruminococcus, associated with fat intake, and Streptococcus, associated with dairy intake, were positively associated with omnivore diets, while a higher level of colonic 
deamination products was found in subjects with low adherence to MD [16]. L-Ruminococcus, which belongs to the Lachnospiracae family, has been found in subjects with low levels of physical activity and Streptococcus is highly abundant in overweight/ obese individuals and those with a higher BMI [17]. De Filippis, et al., uncovered a gradient stratifying bacterium on the basis of diet and microbiota, as well as a positive correlation between MD adherence and the HFD [16].

In a separate study examining shifts on gut microbiota associated with MD adherence, GarciaMantrana et al., found associations between body weight, bacterial groups and observation of MD. Similar to the observations reported by De Filippis et al., a higher MD adherence score was shown to be related to a greater presence of Bacteroidetes [2]. Individuals who exhibited a higher MD adherence score were also observed to have significantly higher fiber intake, compared with those participants in the lower MD score group [2]. In agreement with the aforementioned study, Garcia-Mantrana and colleagues found that subjects with a higher intake of animal protein, saturated fat and/or simple sugars (i.e., Western Diet) demonstrated a significantly higher ratio of Firmicute: Bacteroidetes while simultaneously lower microbial richness/diversity [2]. Mantrana and colleagues also discovered a significant association between MD adherence and elevated Christensenellaceae levels, which have previously been associated in greater presence with lean subjects compared to obese individuals. Interestingly, adolescents and adults with IBD often demonstrate lower levels of Christensenellaceae, hence suggesting the potential for this genus to serve as a pathogenicity predictive biomarker. Finally, upon collection of fecal samples, individuals with a higher MD adherence score indeed exhibited a higher enrichment of both Bifidobacterium and Lactobacillus ( $L A B$ ). Both genera of these bacteria produce lactic acid as a product of lactose fermentation, as well as that of glucose and other sugars [2]. Additionally, Bifidobacterium and $L A B$ act to stimulate the growth of other bacterial communities which produce butyrate and methane as fermentation by-products of fiber in the colon $[2,4]$.

Luisi et al., at the Don Gnocchi Foundation in Florence, Italy, and Francesca Galle from the University of Naples, further explored the role that $L A B$ play in regard to gut health. This is of particular interest, since only recently was $\angle A B$ recognized as a probiotic agent due to its antioxidant/nutraceutical properties and role in protein production, mucosa-bacterial adhesion promotion, and immune cell stimulation in response to gut stimuli [18]. Luisi and colleagues assigned overweight and obese individuals to the dependent variable and normal weight individuals to the control. Both participant groups were administered MD, enriched with $40 \mathrm{~g} /$ day high-quality extra virgin olive oil (HQ-EVOO) over a 3-month period [18]. At the end of the study, the presence of $L A B$ populations assessed demonstrated a significant increase compared to baseline measures in both groups, however the effect was greater for those in the overweight/obese group than in normal weight subjects [18]. Galle et al., also reported similar correlation between $M D$ adherence and $L A B$ proliferation [17]. Both research groups independently suggest that their findings are largely attributed to the generous supplementation of HQ-EVOO. Olive oil is a rich source of dietary polyphenols which both studies demonstrated to contribute significantly in the gut microbiome maintenance. By regulating oxidative status of the intestinal barrier, olive oil may well serve to aid with inflammatory and immune responses of the gut, hence supporting previous research suggesting that olive oil holds prebiotic properties [17, 18].

The previously named microorganisms associated with the MD play a crucial role in altering or establishing a healthy gut microbiome. As a result, adherence to MD has been shown to have 
positive effects on gastrointestinal diseases, including but not limited to certain GI related cancers, inflammatory bowel disease (IBD), irritable bowel syndrome (IBS), Crohn's disease and ulcerative colitis (UC). Recent clinical and epidemiological studies have recorded an improvement or complete resolution of symptoms associated with such diseases by following MD. Table 1 below reports the results of recently conducted studies which explored the relationship between functional gastrointestinal disorders (FGID) and MD. The mechanism by which MD is believed to be associated with reduced irritation, caused by FGID symptoms, has been attributed to the individual food constituents which collectively and individually have the ability to modulate gut inflammation [19].

Table 1 Studies recording possible benefits of MD on gastrointestinal diseases.

\begin{tabular}{lllll}
\hline Reference & Subjects & Treatment & Outcome \\
\hline Akakidis et & 1,116 elementary & FGID identification and Good adherence to MD \\
al. [20] & and high school adherence to MD was reduces risk of developing \\
& students; & assessed using & 2 & FGIDs among children and in \\
& Prospective cohort questionnaires & & adolescents.
\end{tabular}

Khalili et al. 83,147 Swedish A validated FFQ was used to

Adherence to MD is [21] adults; Prospective calculate (modified) MD associated with later-onset adherence at baseline and of CD, but not UC. then assessed for CD and UC onset 17 years post

Papada et 86 patients with CD; Medical history, diseases al. [22] Clinical trial activity, dietary intake, MD adherence score, improved quality of life in CD anthropometric measures patients. and IBDQ were recorded

Chicco et 142 patients with Patients with both CD and UC Short-term (6 mo.) MD al. [23] IBD; Clinical trial followed MD for 6 moths. adherence showed trend towards "normalization" of gut microbiota and a significant reduction in liver steatosis among IBD patients. 


\begin{tabular}{llll}
\hline $\begin{array}{l}\text { Godney et } 153 \text { patients with } \\
\text { al. [24] }\end{array}$ & $\begin{array}{l}\text { Patients who received pouch In patients who had } \\
\text { IBD; Clinical trial }\end{array}$ & $\begin{array}{l}\text { surgery due to UC were undergone pouch surgery, } \\
\text { assessed on their MD adherence to MD was } \\
\text { adherence and pouch associated with decreased } \\
\text { behavior or pouch disease fecal calprotectin levels, } \\
\text { activity. }\end{array}$ & which is used as an indicator \\
& & for intestinal inflammation.
\end{tabular}

Table 1 Crohn's Disease (CD); Food Frequency Questionnaire (FFQ); Functional Gastrointestinal Disease (FGID); Inflammatory Bowel Disease Questionnaire (IBDQ); Ulcerative Colitis (UC).

\section{Mediterranean Diet-Specific Nutrients and Bioactive Compounds}

The Mediterranean diet is becoming increasingly more popular due to growing evidence regarding the role it can play in chronic disease prevention and immune response modulation [5]. Characterized by a diet rich in fruits, vegetables, fish, olive oil, wine and refined cereal products, the foods that are part of MD are typically rich in fiber, omega-3 fatty acids, polyphenols, SCFAs, PUFA's, phytosterols and antioxidants. Together, these bioactive compounds collectively and synergistically extend anti-inflammatory and anti-oxidative properties which may be needed in counteracting accumulation of Trimethylamine $\mathrm{N}$-oxides (TMAO) and the endotoxin Lipopolysaccharides (LPS).

TMAOs are metabolites produced by bacteria in the liver from the breakdown of choline and carnitine which enhance the accumulation of cholesterol in macrophage foam cells and thus simultaneously promote plaque progression[25, 26]. For this reason, TMAOs are said to be proatherogenic and associated with the development of CVD [27]. In a study of Apoe ${ }^{-/}$mice, over an 8-week period, mice fed TMAOs compared with those who were not saw greater macrophage recruitment, as well as enhanced expression of tumor necrosis factor $\alpha$ (TNF- $\alpha$ ), Interleukin 6 (IL-6) and Intracellular Adhesion Molecule 1 (ICAM1) [28]. Additionally, expression of CD36, a scavenger protein that serves to take up long fatty acid chains, and foam cell formation induced by LDL particles were also enhanced by TMAOs [28]. In a study by the Schulich School of Medicine and Dentistry in London, TMAO levels proved to be diet-dependent among humans: when carnitine was given to meat-eaters, TMAOs were created, contrary to when carnitine was given to vegans, TMAOs did not form. This further supports the argument that diet modification, specifically in favor of MD, can be protective against CVD and function as a risk attenuating practice in a context of medical nutrition therapy [25].

Lipopolysaccharides (LPS) constitute another diet-dependent factor shown to induce low grade systemic inflammation [29]. This class of compounds make up a major cell-wall constituent of gram-negative bacteria and cause endothelial activation by way of a receptor complex consisting of Toll-like receptor 4 (TLR4), CD14 and Myeloid Differentiation factor 2 (MD2) [30]. Endothelial activation is characterized as a proinflammatory state during which the expression of leukocyte adhesion molecules increases, vascular integrity is lost, cytokine production increases and human leukocyte antigen (HLA) production is upregulated. It is commonly noted in the early stages of atherosclerosis and in sepsis [30, 31]. When the Gl microbiome is in an inflammatory state, metabolic endotoxemia occurs and bacterial LPS circulation increases by 2-to-3 fold [4]. Therefore, 
a diet high in saturated fat may further encourage a state of inflammation by upregulating LPS transport, while a diet high in fiber may decrease the proportion of gram-negative bacteria and subsequently prevent endotoxemia, thus contributing to an overall healthier gut environment [4].

\subsection{Fiber}

Dietary fiber can be subdivided into 5 groups: non-starch polysaccharides, resistant starch, resistant oligosaccharides, soluble fiber, and insoluble fiber [13]. Insoluble fiber, such as cellulose and hemi-cellulose, is passed quickly through the stomach and small intestine with little to no digestion at all. Upon reaching the large intestine, the insoluble fiber's main purpose is to serve as a bulking agent for fecal matter, and the local bacteria ferment any remaining carbohydrates to produce a minute quantity of SCFAs [13]. In addition, insoluble dietary fiber blocks LPS translocation and therefore serves to block intestinal endotoxemia [4]. By contrast, soluble fiber is fermented by the gut microbes beginning in the stomach to produce a large amount of SCFA metabolites and said to have prebiotic properties because of its ability to induce "beneficial" bacteria proliferation in the gut [13]. Soluble fiber easily dissolves in water to form a gel-like substance in the large intestine [13]. Both forms are crucial in the fostering of a heathy microbiome. In general, high-fiber diets have been known to decrease the proportion of gramnegative bacteria which are commonly associated with the state of gut dysbiosis [32], improve gut barrier function and enhance microbial richness, while low microbial diversity and wealth is associated with higher adiposity, insulin resistance, dyslipidemia and inflammation [33]. The MD is especially rich in fiber when compared alongside other diet and lifestyles, with the primary source of insoluble fiber coming from vegetables, cereals and grains, and soluble fiber from beans, nuts and fruits [13].

The American Heart Association recommends an Adequate Intake for dietary fiber of 25 to $38 \mathrm{~g}$ per day. However, a study by the medical University of South Carolina suggests that American adults consumed approximately half, at $15.9 \mathrm{~g}$ per day in the year from 2007 to 2008 [34]. This finding is indicative of the potential that a majority of adults may be compromising their gut health and overall well-being as related to optimal fiber intake [34].

At UC Davis, Zhu et al., at conducted a study in which participants consumed MD for 4 days and a fast food (FF) diet for 4 days, in random order, with a 4-day washout period in-between [27]. Total fiber intake of MD at 58.0 ( \pm 8.6) $\mathrm{g} / \mathrm{d}$ was significantly higher compared to the FF diet at 12.9 $( \pm 2.8) \mathrm{g} / \mathrm{d}$. Zhu and colleagues utilized the FF diet as an extreme representation/proxy of the Western diet. Their conclusions support existing evidence that MD fosters enhanced GI composition and microbial function thus favoring a healthier gut.

\subsection{Omega-3 Fatty Acids}

Current research efforts suggest that humans today consuming the Western diet are concerningly deficient in omega-3 fatty acids [35]. In the last two decades, omega-3 fats have gained significant popularity due to their touted benefits on the cardiovascular system, blood pressure, triglyceride levels and gut health [35]. Their most well-known function is to serve as important components of phospholipids which comprise nearly all cell membranes in the human body. Additionally, they have demonstrated the ability to limit platelet aggregation, reduce blood viscosity and fibrinogen production, and increase erythrocyte deformability, thus leading to a 
minimized tendency to form a thrombus [36]. They have been known to lower LDL cholesterol and serum triglycerides, and sometimes have attributed to a slight increase in HDL. Lastly, involved with the many anti-inflammatory pathways, as mentioned previously, the omega- 3 fatty acids work by inhibiting free-radical production [35].

The benefits associated with omega-3 fat consumption lie within their structure. They are essential polyunsaturated fats-the body cannot synthesize them endogenously, therefore they must be acquired from the diet - distinguished from saturated and monounsaturated fats by the presence of two or more double bonds within the fatty-acid chain [37]. The omega-3 fats consist of long chains of Carbon atoms with a Carboxyl group on the $\mathrm{N}$-terminus and a methyl group on the C-terminus. It is their unique long and flexible structures which allows for better passage of nutrients and waste products through the cell membranes [37]. Additionally, the byproducts created by their hydrolysis are used to form eicosanoids, important signaling molecules with a wide array of functions that directly affect the cardiovascular, pulmonary, immune and endocrine systems in the body [37].

The three main kinds of omega-3 fatty acids, alpha-linolenic acid (ALA), docosahexaenoic acid (DHA), and eicosapentaenoic acid (EPA), are a source of lipid that researchers believe may delay and thus enhance the ease of gastric emptying, more so compared with saturated fatty acids [35]. ALA is also noted as C18:3n-3 as it is an 18 Carbon molecule with 3 double bonds, thus classifying it as a medium chain. Meanwhile, EPA (C20: 5n-3) and DHA (C22: 6n-3) are considered long-chain omega-3's [37]. Interestingly, ALA can be converted into EPA and DHA in the liver, however, only in very limited quantities, therefore they still must be acquired from the diet [37]. DHA is of particular importance among the three as it is especially present in the brain. The supplementation of EPA and DHA in human trials has been used in the management of cardiovascular disease, rheumatic arthritis, Alzheimer's disease, dementia, depression, inflammatory bowel disease, ulcerative colitis, hyperlipidemia and psoriasis and cancer prevention $[36,37]$. The exact mechanism by which omega-3 fatty acids are able to alleviate symptoms is difficult to pinpoint and remains a focus of research efforts today. The most significant dietary contributors of omega-3 fatty acids in MD are from olive oil (ALA) and fatty fish such as sardines (DHA and EPA) [35].

\subsection{Polyphenols}

Polyphenols are naturally occurring compounds abundant in plants, namely in acidic fruits and vegetables, involved in the defense system against pathogens and ultraviolent light [38]. When consumed by humans, they have shown a similar defense system against chronic disease. There are over 8,000 polyphenols that have been identified to date, which have been divided among 4 broadly-defined categories: Phenolic acids, flavonoids, stilbenes, and lignans [38]. These compounds are unique due to their antioxidant and protective capacity, in addition to their chemical nature; They can easily accept any free-floating electrons, thereby stabilizing the system, and stopping a free radical from oxidizing another reaction [38, 39]. As a result, their long-term consumption has been shown to have protective effects on many diseases. Against neurodegenerative diseases, polyphenols serve as antioxidants, protect neurons and inhibit the accumulation of beta-amyloid plaques associated with Alzheimer's disease [39]. In cancer, flavonoids can neutralize the free radicals which cause cancer to arise, and arrest growth in tumor 
cells. In cardiovascular health, certain polyphenols assist with ventricular function, platelet activity, anti-inflammatory effects and reduce blood pressure. Finally, in type 2 diabetes, the antiinflammatory and antioxidant properties of polyphenols protect the pancreatic beta-cells from glucose toxicity by assisting with carbohydrate metabolism, thereby enhancing glycemic control. These benefits extend beyond the diseases detailed prior, extending from obesity, to pancreatitis, asthma, Severe Acute Respiratory Syndrome (SARS) and osteoporosis [38, 40]. Lastly, polyphenols have been studied extensively for their ability to aid in anti-aging processes [39].

Tresserra-Rimbau and colleagues at the University of Barcelona, Spain, explored the inverse relationship between habitual polyphenol intake and incidence of cardiovascular disease [10]. Participants were randomized into one of three diet regimen groups: 1) MD supplemented with extra virgin olive oil, 2) MD supplemented with nuts, or 3) the control diet (low-fat). Upon completion of the study, after one year, a greater intake of polyphenols (olive oil) was inversely associated with hypertension and resulted in a $46 \%$ reduction in risk of major cardiovascular events. This study supports current evidence that polyphenols may be protective against CVD and have a health-enhancing/promoting effect by inhibiting inflammatory pathways and improving endothelial function [10, 41].

\subsection{Short Chain Fatty Acids}

Short chain fatty acids (SCFAs) are crucial structures for the integrity and maintenance of the colon [42]. The anaerobic fermentation of undigested or unabsorbed carbohydrate, specifically insoluble fiber, by colonic bacteria produce SCFAs and Hydrogen gas as byproducts; the SCFAs are then absorbed simultaneously with sodium and water in the large intestine while the Hydrogen gas can cause the production of flatulence [42]. SCFAs are considered a subgroup of saturated fatty acids, arranged by 1-6 Carbon atoms [42]. The three most studied SCFAs include acetate, butyrate, and propionate, each differing only slightly in structure but serving unique functions. Nonetheless, Butyrate is more readily metabolized and thus used preferentially over Propionate and Acetate [42].

Current research efforts are aimed at investigating the potential therapeutic effect of SCFAs and their role in iron transport, providing nutrients for the colonic epithelium, as modulators of intracellular $\mathrm{pH}$ and cell volume, and their potential ability to regulate gene expression and differentiation $[42,43]$. Additionally, ongoing clinical research is exploring the potential curative role SCFAs may play in ulcerative colitis and antibiotic-associated diarrhea. Finally, and most notably, SCFAs may be a significant contender in the prevention of colon cancer. The exact mechanism is unclear; however, theories propose that SCFAs are able to induce apoptosis of carcinomic cell lines [42]. The diets highest in fiber and complex carbohydrates, such as the MD and other plant-based diets, are associated with increased SCFA production and accompanying benefits. Notably, a study by the University of Naples demonstrated that even in individuals who typically consume an omnivore diet, a higher adherence to MD correlated with a proportionate increase in fecal SCFA levels [16].

\subsection{Phytosterols}

Phytosterols are plant-derived compounds whose structure and function closely resembles that of cholesterol molecules derived from mammalian food sources [44]. As such, their cholesterol- 
lowering properties have become of great interest to scientists and nutritionists alike, especially among those whose emphases are in Type 2 diabetes and dyslipidemia [44]. Phytosterols and cholesterol share an identical ring structure and differ only in their side chains at the 24 Carbon position of an unsaturated fatty acid [45]. Classified as triterpene hydrocarbons, phytosterols are also defined by their 28-29 Carbon structure, with a methyl group ground on the fourth Carbon atom [45]. It is believed that their likeness to that of cholesterol is the reason why they can have lipid-lowering effects on LDL-cholesterol levels [45, 46].

In a literature review conducted by the Washington University School of Medicine, food frequency questionnaires revealed that extreme consumption of plant sterols may be associated with a 3-14\% reduction in LDL-cholesterol levels [46]. Their consumption was shown to have an effect on cholesterol absorption efficiency, biosynthesis and excretion [46]. Additionally, Jennifer Malinowski, Pharm. D. with the Nesbitt College of Pharmacy and Nursing et al. reviewed over 40 clinical trials and determined that incorporation of 2 grams of phytosterols daily through the diet (margarine, mayonnaise, orange juice, etc.) produced an average of $10-11 \%$ reduction in LDL cholesterol [45]. Further research is necessary to determine if the effects are lasting beyond twoyears [45]. Phytosterols are particularly abundant in the MD compared with other diets due to the emphasis placed on fruit, vegetable, and olive oil intake [44].

\subsection{Antioxidants}

One of, if not the most notable and overarching properties of the food consumed in the MD is the antioxidant capacity. Antioxidants can be most simply described as "man-made or natural substances that may prevent or delay some type of cell damage [47]." Their structure is impossible to outline as unlike the previously named bioactive compounds, it is their function which defines them. Many compounds can be coined 'antioxidant' in nature, varying from fruits and vegetables to olive oil to wine [48]. In all of these named foods (and beverage), molecules are present which can combat the effects of reactive oxygen species (ROS) [48]. ROS, also known as free radicals, are the molecules which cause oxidative cell damage and the subsequent detrimental effects seen in human diseases; They may be endogenous or exogenous such as from radiation [48]. Higher amounts of ROS have consistently been recorded in association with human diseases, including but not limited to arthritis, amyotrophic lateral sclerosis, cancer, diabetes, atherosclerosis, ischemia and failures in immunity and endocrine function [48]. Thus, the lower the total concentration of ROS in the body, the more enhanced the body is at defending against pathogens and in regulating intracellular signaling [48]. Antioxidants function to neutralize free radicals and protect the body by maintaining a "redox balance" [48].

In a study by the School of Medicine in Athens, Greece, 3,042 individuals who had no clinical evidence of cardiovascular disease were assessed on their MD adherence and immunodiagnostic assay [49]. Researchers found that the greater a participant adhered to the MD, the greater their total antioxidant capacity (TAC) score [49]. Participants in the highest tertile of MD adherence had, on average, $11 \%$ higher antioxidant levels than did participants in the lowest tertile; Additionally, the same group who saw the elevated TAC score, also saw a 19\% lower oxidized LDL concentration, when compared to those in the lower tertile [49]. This study served to support the amounting evidence that the MD is indeed antioxidant, and thus anti-inflammatory and anticarcinogenic as well, in nature, in addition to boasting significant benefits on cardiovascular health. 


\section{The Role of the Mediterranean Diet in Pathophysiology of Chronic Diseases}

The protective benefits and potential to treat certain chronic illnesses through implementation of MD, namely cardiovascular disease, gastrointestinal diseases, Alzheimer's and atherosclerosis are the focal points of many researchers.

In 2019, the American Heart Association publicly stated that a Mediterranean-style diet has indeed demonstrated impressive cardioprotective effects [50]. In addition, a study conducted by Estruch et al., concluded that individuals on MD supplemented with either EVOO or nuts, exhibited a lower incidence of cardiovascular event(s) than those on a reduced-fat diet [51]. This cardioprotective effect can largely be attributed to the nature/characteristics of the dietary scheme: rich in omega- 3 fatty acids and antioxidant provision from the abundance of fruits and vegetables.

The extent to which gut health impacts general health status is still largely unknown. Researchers are merely beginning to delve into the underlying reasons behind gastrointestinal disorders, but several observations support that the status of the gut microbiome is significantly involved. Zito and colleagues at the University of Naples sought to evaluate how elevated adherence to the MD could prevent GI symptoms [35]. The researchers discovered that MD adherence scores were significantly lower in subjects who regularly complained of GI symptoms, than those who had none, therefore, researchers proposed that an inverse relationship might plausibly exist. Zito et al., suggest that imbalanced diets may contribute to the phenotype that functional dyspepsia and irritable bowel syndrome present. Further research is necessary to determine if following a plant-based diet, consistently over an extended period of time, can ameliorate GI symptoms [35].

In the Department of Internal Medicine-Molecular Medicine at Winston-Salem, North Carolina, Nagpal et al., conducted a study evaluating how a modified Mediterranean-ketogenic diet (MMKD) can modulate the gut microbiome and potentially detect early onset of Alzheimer's disease [29]. Nagpal et al., concluded that subjects with impaired cognition have noticeably unique microbial population profile signatures in comparison to those with normal cognition, suggesting the potential utility of gut biomarkers for neurogenerative disease detection [29]. Researchers also found that the abundance of certain bacteria genera is reduced with MMKD and that subjects on MMKD recorded reduced fecal lactate and acetate levels, while propionate and butyrate were recorded in greater quantities than at baseline [29].

Atherosclerosis, characterized by the accumulation of plaque inside the artery walls, is a condition nearly entirely dependent on dietary factors, but beyond solely fat content of the diet. As discussed previously, the proliferation of TMAOs is pro-atherogenic, while TMAO is the product from the bacterial breakdown of choline and carnitine, which are sourced from animal products. Subsequently, their circulation induces systemic inflammation thus increasing predisposition towards cardiovascular diseases. For this reason, MD and plant-based based diets have received acclaim for being anti-inflammatory and inhibitory against chronic disease [25]. There is evidence that best practices can improve risk and/or management of chronic diseases such as Type 2 Diabetes Mellitus [40,52]. In this context, Mediterranean Diet can function as a best practice for risk attenuation in a framework of medical nutrition therapy and lifestyle optimization. Finally, an important point regarding the Mediterranean Diet is that it is regarded as a lifestyle practice which extends beyond a mere dietary scheme [53]. Other elements pertinent to lifestyle including 
physical activity, social companionship and bonding as well as stress management all seem to be significant additional contributors to the health benefits extended by the Mediterranean Diet.

\section{Conclusions}

The aim of this brief review was to briefly outline the effects of MD on the gut microbiome and subsequently on health status from a clinical point of view based on recent evidence from human studies. The Mediterranean Diet is characterized by its emphasis placed onto fruit, vegetable, unsaturated fat, and moderate alcohol consumption, as well as its rich content of fiber, omega-3 fatty acids, polyphenols, short chain fatty acids, phytosterols and antioxidants. The Mediterranean diet is now recommended by practitioners and health professionals alike due to its protective benefits against many chronic illnesses and metabolic diseases in the context of best practice and medical nutrition therapy. Further research is needed to determine which metabolic diseases MD is protective against, through what precise mechanisms, and to what extent overall health depends on gut microbiome status.

\section{Acknowledgments}

The authors would like to extend their thanks to Dr. Aleksandra S. Kristo, faculty in the Food Science and Nutrition Department of California Polytechnic State University, for her constructive input on the manuscript.

\section{Author Contributions}

AKS conceived the idea and designed the manuscript's framework. AKS and AHK performed literature search. AKS and AHK co-wrote the manuscript.

\section{Funding}

This research did not receive any specific grant from funding agencies in the public, commercial, or not-for-profit sectors.

\section{Competing Interests}

The authors have declared that no competing interests exist.

\section{References}

1 Sikalidis AK, Maykish A. The gut microbiome and type 2 diabetes mellitus: Discussing a complex relationship. Biomedicines. 2020; 8: 8.

2 Garcia-Mantrana I, Selma-Royo M, Alcantara C, Collado MC. Shifts on gut microbiota associated to mediterranean diet adherence and specific dietary intakes on general adult population. Front Microbiol. 2018; 9: 890.

3 Katz DL, Meller S. Can we say what diet is best for health? Annu Rev Public Health. 2014; 35: 83-103.

4 Bailey MA, Holscher HD. Microbiome-mediated effects of the Mediterranean diet on inflammation. Adv Nutr. 2018; 9: 193-206. 
5 Meslier V, Laiola M, Roager HM, De Filippis F, Roume H, Quinquis B, et al. Mediterranean diet intervention in overweight and obese subjects lowers plasma cholesterol and causes changes in the gut microbiome and metabolome independently of energy intake. Gut. 2020; 69: 12581268.

6 Rouen PA, Wallace BR. The 2015-2020 dietary guidelines: Overview and implications for nursing practice. Home Healthc Now. 2017; 35: 72-82.

7 Nagpal R, Shively CA, Register TC, Craft S, Yadav H. Gut microbiome-Mediterranean diet interactions in improving host health. F1000research. 2019; 8: 699.

8 Robertson MD, Jackson KG, Fielding BA, Morgan LM, Williams CM, Frayn KN. Acute ingestion of a meal rich in $\mathrm{n}-3$ polyunsaturated fatty acids results in rapid gastric emptying in humans. Am J Clin Nutr. 2002; 76: 232-238.

9 Cassidy A, Mukamal KJ, Liu L, Franz M, Eliassen AH, Rimm EB. High anthocyanin intake is associated with a reduced risk of myocardial infarction in young and middle-aged women. Circulation. 2013; 127: 188-196.

10 Tresserra-Rimbau A, Rimm EB, Medina-Remón A, Martínez-González MA, De la Torre R, Corella $D$, et al. Inverse association between habitual polyphenol intake and incidence of cardiovascular events in the PREDIMED study. Nutr Metab Cardiovasc Dis. 2014; 24:639-647.

11 Zinöcker MK, Lindseth IA. The Western diet-microbiome-host interaction and its role in metabolic disease. Nutrients. 2018; 10: 365.

12 Sonnenburg ED, Sonnenburg JL. Starving our microbial self: The deleterious consequences of a diet deficient in microbiota-accessible carbohydrates. Cell Metab. 2014; 20: 779-786.

13 Makki K, Deehan EC, Walter J, Bäckhed F. The impact of dietary fiber on gut microbiota in host health and disease. Cell Host Microbe. 2018; 23: 705-715.

14 Hales CM, Carroll MD, Fryar CD, Ogden CL. Prevalence of obesity among adults and youth: United States, 2015-2016. NCHS Data Brief. 2017; 288: 1-8.

15 Peterson J, Garges S, Giovanni M, Mclnnes P, Wang L, Schloss JA, et al. The NIH human microbiome project. Genome Res. 2009; 19: 2317-2123.

16 De Filippis F, Pellegrini N, Vannini L, Jeffery IB, La Storia A, Laghi L, et al. High-level adherence to a Mediterranean diet beneficially impacts the gut microbiota and associated metabolome. Gut. 2016; 65: 1812-1821.

17 Gallè F, Valeriani F, Cattaruzza MS, Gianfranceschi G, Liguori R, Antinozzi M, et al. Mediterranean diet, physical activity and gut microbiome composition: A cross-sectional study among healthy young Italian adults. Nutrients. 2020; 12: 2164.

18 Luisi ML, Lucarini L, Biffi B, Rafanelli E, Pietramellara G, Durante M, et al. Effect of mediterranean diet enriched in high quality extra virgin olive oil on oxidative stress, inflammation and gut microbiota in obese and normal weight adult subjects. Front Pharmacol. 2019; 10: 1366.

19 Vrdoljak J, Vilović M, Živković PM, Tadin Hadjina I, Rušić D, Bukić J, et al. Mediterranean diet adherence and dietary attitudes in patients with inflammatory bowel disease. Nutrients. 2020; 12: 3429.

20 Agakidis C, Kotzakioulafi E, Petridis D, Apostolidou K, Karagiozoglou-Lampoudi T. Mediterranean diet adherence is associated with lower prevalence of functional gastrointestinal disorders in children and adolescents. Nutrients. 2019; 11: 1283.

21 Khalili H, Håkansson N, Chan SS, Chen Y, Lochhead P, Ludvigsson JF, et al. Adherence to a 
Mediterranean diet is associated with a lower risk of later-onset Crohn's disease: Results from two large prospective cohort studies. Gut. 2020; 69: 1637-1644.

22 Papada E, Amerikanou C, Forbes A, Kaliora AC. Adherence to Mediterranean diet in Crohn's disease. Eur J Nutr. 2020; 59: 1115-1121.

23 Chicco F, Magrì S, Cingolani A, Paduano D, Pesenti M, Zara F, et al. Multidimensional impact of Mediterranean diet on IBD patients. Inflamm Bowel Dis. 2021; 27: 1-9.

24 Godny L, Reshef L, Pfeffer-Gik T, Goren I, Yanai H, Tulchinsky H, et al. Adherence to the Mediterranean diet is associated with decreased fecal calprotectin in patients with ulcerative colitis after pouch surgery. Eur J Nutr. 2020; 59: 3183-3190.

25 Pignanelli M, Just C, Bogiatzi C, Dinculescu V, Gloor GB, Allen-Vercoe E, et al. Mediterranean diet score: Associations with metabolic products of the intestinal microbiome, carotid plaque burden, and renal function. Nutrients. 2018; 10: 779.

26 Trøseid M, Hov JR, Nestvold TK, Thoresen H, Berge RK, Svardal A, et al. Major increase in microbiota-dependent proatherogenic metabolite TMAO one year after bariatric surgery. Metab Syndr Relat Disord. 2016; 14: 197-201.

27 Zhu C, Sawrey-Kubicek L, Beals E, Rhodes CH, Houts HE, Sacchi R, et al. Human gut microbiome composition and tryptophan metabolites were changed differently by fast food and Mediterranean diet in 4 days: A pilot study. Nutr Res. 2020; 77: 62-72.

28 Geng J, Yang C, Wang B, Zhang X, Hu T, Gu Y, et al. Trimethylamine N-oxide promotes atherosclerosis via CD36-dependent MAPK/JNK pathway. Biomed Pharmacother. 2018; 97 : 941-947.

29 Nagpal R, Neth BJ, Wang S, Craft S, Yadav H. Modified Mediterranean-ketogenic diet modulates gut microbiome and short-chain fatty acids in association with Alzheimer's disease markers in subjects with mild cognitive impairment. EBioMedicine. 2019; 47: 529-542.

30 Dauphinee SM, Karsan A. Lipopolysaccharide signaling in endothelial cells. Lab Invest. 2006; 86: 9-22.

31 Hunt BJ, Jurd KM. Endothelial cell activation: A central pathophysiological process. BMJ. 1998; 316: 1328-1329.

32 Weickert MO, Pfeiffer AF. Metabolic effects of dietary fiber consumption and prevention of diabetes. J Nutr. 2008; 138: 439-442.

33 Le Chatelier E, Nielsen T, Qin J, Prifti E, Hildebrand F, Falony G, et al. Richness of human gut microbiome correlates with metabolic markers. Nature. 2013; 500: 541-546.

34 King DE, Mainous III AG, Lambourne CA. Trends in dietary fiber intake in the United States, 1999-2008. J Acad Nutr Diet. 2012; 112: 642-648.

35 Zito FP, Polese B, Vozzella L, Gala A, Genovese D, Verlezza V, et al. Good adherence to mediterranean diet can prevent gastrointestinal symptoms: A survey from Southern Italy. World J Gastrointest Pharmacol Ther. 2016; 7: 564-571.

36 Simopoulos AP. Omega-3 fatty acids in health and disease and in growth and development. Am J Clin Nutr. 1991; 54: 438-463.

37 Office of Dietary Supplements. Omega-3 fatty acids [Internet]. Bethesda, MD: National Institutes of Health; $2005 . \quad$ Available from: https://ods.od.nih.gov/factsheets/Omega3Fatty\%20AcidsandHealth-HealthProfessional/.

38 Pandey KB, Rizvi SI. Plant polyphenols as dietary antioxidants in human health and disease. Oxid Med Cell Longev. 2009; 2: 270-278. 
39 Cory H, Passarelli S, Szeto J, Tamez M, Mattei J. The role of polyphenols in human health and food systems: A mini-review. Front Nutr. 2018; 5: 87.

40 Sikalidis AK, ÖZTAĞ M. Optimized snacking is positively associated with socioeconomic status and better type 2 diabetes mellitus management in Turkish patients. Gazz Med Ital Arch Sci Med. 2020; 179: 459-467.

41 Tuttolomondo A, Simonetta I, Daidone M, Mogavero A, Ortello A, Pinto A. Metabolic and vascular effect of the Mediterranean diet. Int J Mol Sci. 2019; 20: 4716.

42 Tan J, McKenzie C, Potamitis M, Thorburn AN, Mackay CR, Macia L. The role of short-chain fatty acids in health and disease. Adv Immunol. 2014; 121: 91-119.

43 Cook SI, Sellin JH. Short chain fatty acids in health and disease. Aliment Pharmacol Ther. 1998; 12: 499-507.

44 Malinowski JM, Gehret MM. Phytosterols for dyslipidemia. Am J Health Syst Pharm. 2010; 67: 1165-1173.

45 Dumolt JH, Rideout TC. The lipid-lowering effects and associated mechanisms of dietary phytosterol supplementation. Curr Pharm Des. 2017; 23: 5077-5585.

46 Racette SB, Lin X, Ma L, Ostlund Jr RE. Natural dietary phytosterols. J AOAC Int. 2015; 98: 679684.

47 National Center for Complementary and Integrative Health. Antioxidants: In Depth [Internet]. Bethesda, MD: National Institutes of Health; 2013. Available from: https://www.nccih.nih.gov/health/antioxidants-in-depth.

48 Rajendran P, Nandakumar N, Rengarajan T, Palaniswami R, Gnanadhas EN, Lakshminarasaiah U, et al. Antioxidants and human diseases. Clin Chim Acta. 2014; 436: 332-347.

49 Pitsavos C, Panagiotakos DB, Tzima N, Chrysohoou C, Economou M, Zampelas A, et al. Adherence to the Mediterranean diet is associated with total antioxidant capacity in healthy adults: The ATTICA study. Am J Clin Nutr. 2005; 82: 694-699.

50 Singh RB, Dubnov G, Niaz MA, Ghosh S, Singh R, Rastogi SS, et al. Effect of an IndoMediterranean diet on progression of coronary artery disease in high risk patients (IndoMediterranean Diet Heart Study): A randomised single-blind trial. Lancet. 2002; 360: 14551461.

51 Estruch R, Ros E, Salas-Salvadó J, Covas MI, Corella D, Arós F, et al. Primary prevention of cardiovascular disease with a Mediterranean diet supplemented with extra-virgin olive oil or nuts. N Engl J Med. 2018; 378: e34.

52 Sikalidis AK, KARABOĞA EP. Healthy diet and self-care activities adherence improved lifequality and type 2 diabetes mellitus management in Turkish adults. Gazz Med Ital Arch Sci Med. 2020; 179: 528-537.

53 Sikalidis AK. From food for survival to food for personalized optimal health: A historical perspective of how food and nutrition gave rise to nutrigenomics. J Am Coll Nutr. 2019; 38: 8495. 
OBM Hepatology and Gastroenterology 2021; 5(1), doi:10.21926/obm.hg.2101056

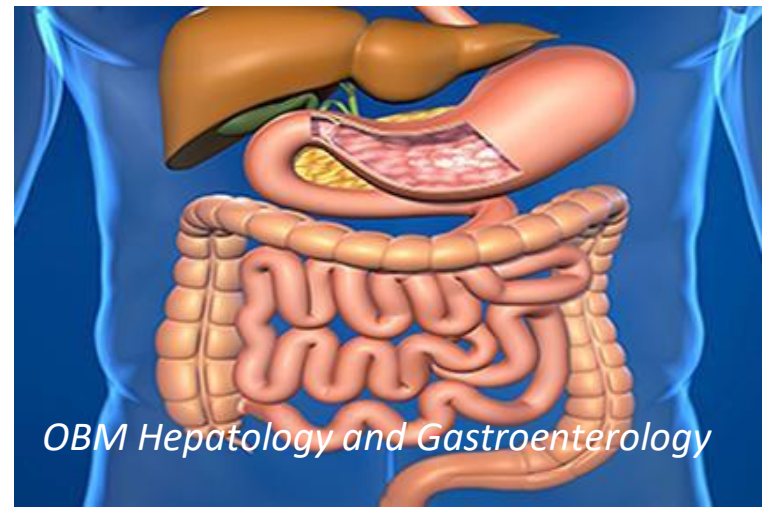

Enjoy OBM Hepatology and Gastroenterology by:

1. Submitting a manuscript

2. Joining in volunteer reviewer bank

3. Joining Editorial Board

4. Guest editing a special issue

For more details, please visit:

http://www.lidsen.com/journals/hg 